\title{
LA PARTE INTRODUCTORIA DEL EPISTOLARIO PAULINO
}

\author{
Silvestre Pongutá H., SDB \\ Pontificia Universidad Javeriana de Bogotá \\ spongutah@yahoo.com
}

Resumen: Los comentarios del epistolario paulino presentan exegéticamente la propia lectura de la praescriptio y de las demás partes de cada carta. Una lectura de las primeras partes (de la praescriptio) no de una, sino de todas las cartas de san Pablo, puede brindar una visión muy completa y llegar a ser una especie de puerta de entrada de los escritos del apóstol. El lector hallará aquí no una exégesis de los textos, sino una aproximación que ayude a valorar los datos que san Pablo brinda de sí mismo en esta primera parte de sus escritos, las indicaciones que da de sus destinatarios y lo principal del contenido de sus saludos y augurios. Para el análisis de esta primera parte se tendrá en cuenta una agrupación muy común de las cartas: las primeras en orden cronológico, las llamadas Cartas de la Cautividad y las Pastorales. Por los límites de este artículo se ha omitido toda discusión y opinión acerca de la autenticidad de algunas de las cartas: solo se desea presentar lo que estos breves textos dicen.

Palabras clave: Cartas antiguas. Retórica. Epistolas paulinas. Nuevo Testamento. Praescriptio.

\section{The Intoductory Part of Paul's Letters}

Abstract: The comments of the epistolary of St. Paul presents the very reading of the Praescriptio and of the other parts of each letter. A reading of the first parts (the Praescriptio) not of a single but of all the letters of St. Paul can provide a very complete vision and become a kind of gateway to the writings of the Apostle. The reader will find here not an exegesis of the 
texts but an approximation that helps to value the data that Saint Paul provides of himself in this first part of his writings, the indications given of his recipients and the main content of his greetings and omens. For the analysis of this first part one will consider a very common grouping of the letters: the first ones in chronological order, the so-called letters of the captivity and the pastoral ones. The limits of this article have omitted any discussion and opinion about the authenticity of some of the letters: you only want to present what these brief texts say.

Key words: Ancient Letters. Rhetorics. Pauline Epistles. New Testament. Praescriptio.

Algo muy característico del conjunto de los escritos del Nuevo Testamento es la notable presencia de epístolas. Entre ellas, las más numerosas son las atribuidas a san Pablo.

Los autores de introducciones al NT, y, en algunos casos, también los comentaristas de este tipo de escritos, suelen relacionar la parte introductoria con textos provenientes de las culturas helenista y grecolatina. A manera de ejemplo, R. E. Brown cita un estudio de A. Deissmann en el que ofrece en primer lugar la clasificación de carta y epístola; posteriormente presenta la estructura propia de estos escritos; a la forma introductoria le da el nombre latino de praescriptio: esta tiene tres subpartes: superscriptio (la persona que envía la epístola o carta, el remitente), la adscriptio (destinatario) y la salutatio (saludo) ${ }^{1}$. La estructura, con los nombres mencionados de sus partes, parecen ser más característicos de las cartas, si bien se pueden constatar también en las epístolas. En las cartas prevalece el aspecto de correspondencia personal; la epístola presenta más una cierta oficialidad, y en ella son importantes los temas que trata. De hecho, las anotaciones de Deissmann son constatables en las epístolas o cartas de san $\mathrm{Pablo}^{2}$, pero

1 Brown, Introduction, 410-412; la cita de A. DeISSMAnN la hace de la traducción inglesa: Light from the Ancient East, London ${ }^{21927 . ~ L a s ~ o t r a s ~ p a r t e s ~ d e ~ l a ~ f o r m a ~ q u e ~}$ reconoce el mismo autor son la acción de gracias, el mensaje central y la forma conclusiva.

2 Se debe reconocer quizá que, en la totalidad de los escritos de san Pablo, quien escribe es el apóstol, perfectamente consciente de su identidad derivada de la llamada del Resucitado y con una intención clara de actuar motivado por la misión recibida del Señor. Desde este punto de vista, sus escritos serían más epístolas que cartas; sin embargo, convencionalmente las podemos mencionar con el nombre cartas, pero sin menoscabar el carácter oficial de las mismas. 
no de una manera estricta: el apóstol usa las características formales de este estilo, y lo hace con una gran libertad.

El presente estudio solo pretende realizar un acercamiento a la parte introductoria de las cartas de san Pablo (la praescriptio). Las anotaciones de Deissmann, asumidas por Brown y seguidas por otros comentaristas de las cartas, son en verdad muy prácticas para el análisis que se intenta compartir con los lectores. Para facilitar la presentación del estudio se hará por orden y de manera separada el análisis de las subpartes de la praescriptio; además, se agruparán las cartas de la siguiente manera:

1.1: 1 Ts; 2 Ts; Gal; 1 Cor; 2 Cor; Rom.

1.2: Flp; Flm; Col; Ef.

1.3: Tit; $1 \mathrm{Tm} ; 2 \mathrm{Tm}$.

\section{La superscriptio}

En los tres grupos de cartas, el remitente se menciona con el nombre de Pablo (Paûlos). Este nombre, como afirma Brown ${ }^{3}$, era bien conocido y empleado como un nombre que indicaba la familia a la que pertenecía la persona; Lucas, en Hch 7,58, al mencionarlo, lo hace con el nombre semita Saulo. Parece que era normal que los judíos que vivían en la diáspora emplearan dos nombres: uno del uso helenista y otro de su pertenencia al pueblo judío. Es también probable que el nombre grecorromano pudiera ser utilizado por quienes habían nacido en ciudades consideradas libres (eran ciudadanos romanos).

La superscriptio de las cartas paulinas no se hace solo con el nombre del remitente. Del conjunto se puede constatar una presentación muy variada y significativa de la comprensión que Pablo tiene de sí mismo y que desea compartir con los destinatarios de sus escritos. Esta comprensión se puede verificar en los términos empleados por el apóstol para acompañar su propio nombre; los principales son: "apóstol" (se lee en todas las cartas menos en 1 y 2 Ts; Flp; Flm); en algunos casos afirma que es "apóstol llamado" (o "apóstol por vocación": 1 Cor 1,1; Rom 1,1); en relación con este término se deben mencionar algunas precisiones o agregados destinados a enriquecer los aspectos que se quieren insinuar; el lector encontrará algunas precisiones al examinar textos pertinentes; algunas presentaciones del remitente poseen una notable extensión (cf. Gal; Rom; Tit). Otro término de la presentación de san Pablo es el de "siervo": "siervo de Cristo Jesús" (Rom

3 Brown, Introduction, 423. 
1,1; Flp 1,1); "siervo de Dios" (Tit 1,1). En la superscriptio de Filemón emplea el término "prisionero" de Cristo Jesús (Flm 1). Cada uno de estos términos tiene, desde luego, su propio significado y su propia función.

Una de las constataciones que llaman la atención del lector es la de ver que el remitente (san Pablo) asocia a otras personas: "Silvano y Timoteo" (1 Ts 1,1; 2 Ts 1,1); "Sóstenes, el hermano" (1 Cor 1,1); "Timoteo, el hermano" (2 Cor 1,1; Flm 1,1; Col 1,1); "todos los hermanos que están conmigo" (Gal 1,1). Este hecho tal vez no permite afirmar una responsabilidad compartida en la redacción de los escritos, pero sí da un colorido comunitario y eclesial a la actividad del apóstol y a la intervención que quiere hacer en las Iglesias a las que dirige sus cartas. Algunos de estos acompañantes tendrán más tarde responsabilidades en la consolidación de la fe o en la dirección de diversas comunidades.

La ampliación de la superscriptio es muy notoria en la carta a los Gálatas, más todavía en la dirigida a la Iglesia de Roma, y también en la carta a Tito.

\subsection{La superscriptio en el primer grupo de cartas (1 Ts; 2 Ts; Gal; 1 Cor; 2 Cor; Rom)}

San Pablo escribió estas cartas en el primer período de su actividad como apóstol (entre los años 51-57 d. C.). Las Iglesias de Tesalónica, las de Galacia y la de Corinto fueron fundadas por el apóstol. Lo peculiar de las cartas a los Tesalonicenses, en la superscriptio, es el hecho de no mencionar explícitamente su condición de apóstol y sí agregar a su nombre los de Silvano y Timoteo. Para san Pablo debió de ser espontáneo pensar que con su solo nombre se tenía clara tanto su relación con Jesucristo como la explicación de su misión.

La superscriptio de la carta a los Gálatas ofrece una considerable ampliación. Por el contexto de la carta se sabe que en las comunidades de Galacia, llegadas a la fe por la evangelización de Pablo y cuyos miembros en su mayoría habían sido paganos, aparecieron algunos problemas suscitados por la intervención de los llamados judaizantes: estos seguían aferrados a todas las prescripciones de la Ley y, por tanto, pretendían que los paganos, antes de ser cristianos, llegaran a ser judíos; para ello exigían la circuncisión y la observancia de los preceptos de la Ley. Esto equivalía a alterar el evangelio, a desvirtuar la persona y la obra de Cristo y a desautorizar la evangelización que san Pablo había realizado en las comunidades de Galacia y seguía realizando en otros lugares. La dureza de algunas 
expresiones que se leen en esta carta documenta la contrariedad sufrida por el apóstol y explica los temas que trata y el modo en que los formula. El texto de la superscriptio es el siguiente:

Pablo, apóstol, no de parte de hombres ni mediante hombre alguno, sino por Jesucristo y Dios, nuestro Padre, quien lo resucitó de los muertos, y todos los hermanos que están conmigo 4 .

Pablo, en cuanto remitente, se presenta en primer plano como apóstol: es lo más importante que habrían debido tener en cuenta los que recibieron directamente la evangelización de san Pablo y ahora son los destinatarios de su carta. La densidad significativa del término estará en relación directa con la fuente de donde procede, es decir, de quien le ha dado este encargo y de lo que implica ${ }^{5}$. La primera precisión que hace san Pablo es la afirmación enfática sobre el origen de su apostolado: no procede de hombres ni siquiera por la mediación de algún hombre. No está claro el motivo que llevó a Pablo a realizar esta primera precisión: una hipótesis puede ser la implícita comparación que se hizo en las comunidades de Galacia de la persona y la obra de Pablo con antiguos profetas o con alguno o algunos de los mismos doce apóstoles, ya que ellos sí conocieron personalmente a Jesús. Al eliminar de la fuente de su apostolado cualquier mediación humana, san Pablo manifiesta de manera explícita que él llegó a ser apóstol directamente por medio de Jesucristo y, en definitiva, todo depende de Dios, nuestro Padre, puesto que fue él quien resucitó a Jesucristo. Para todos debe ser perfectamente clara la íntima, profunda y misteriosa relación de Jesucristo con Dios. Si el Resucitado fue quien eligió, llamó y envió a Pablo, de ahí surge el valor y la decisiva importancia de su apostolado: cuando llegó a las comunidades de Galacia, Pablo lo hizo en representación del mismo Señor resucitado. Esta segunda precisión es entonces la decisiva, mucho más si el Resucitado está revelando y haciendo presente al mismo Dios.

Por otra parte, el término "apóstol" parece muy típico del Nuevo Testamento. En singular se encuentra 18x, de las cuales solo 3x en escritos

${ }^{4}$ El texto que ofrece el programa BibleWorks es: Пaṽ

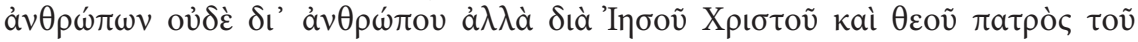

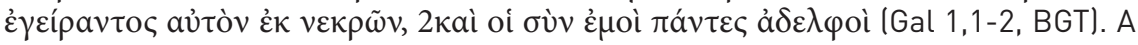
los promotores de este programa llegue nuestra valoración y nuestra gratitud. Este texto coincide con el de la edición $28^{\mathrm{a}}$ de Nestle-Aland.

5 Algunos comentaristas relacionan el término "apóstol" con la palabra hebrea šăliah, presente en un texto de la Misná: "El representante de una persona es como si fuera ella misma” (cf. Misná, Ber. V, 5): cf. Fitzmyer, NCBSJ 2, 292; Schlier, Galati, 28; Mussner, Galater Brief, 46. 
no paulinos (Jn 13,16; 1 Pe 1,1; 2 Pe 1,1). En plural se halla muchas veces, y se refiere al grupo de los doce a partir de los textos de Mc 3,14 y especialmente de Lc 6,13. Este término tiene mucha importancia en la cristología y en la eclesiología. Los doce fueron elegidos directamente por Jesucristo, y fue el Resucitado quien los envió (cf. Mt 28,18-20). Ciertamente, no fue el Jesús de la historia quien eligió a Pablo; él fue elegido por el Señor resucitado y él fue quien lo envió: a pesar de no pertenecer al grupo de los doce, san Pablo, lo mismo que los doce, es también enviado (apóstol).

Agrega, como es su costumbre, la mención de las personas que acompañan a Pablo: en esta carta lo hace de manera genérica: "Todos los hermanos que están conmigo"; Pablo no está solo. Él tiene hermanos que lo acompañan.

En cartas posteriores empleará una fórmula sintética, pero que tiene la misma función de hacer presente a quien da autoridad a la misión del apóstol: "Por voluntad de Dios" (cf. 1 y 2 Cor; Col; Ef; 2 Tm; en 1 Tm se lee una expresión distinta, pero equivalente: "Según el mandato de Dios, Salvador nuestro, y de Cristo Jesús, esperanza nuestra") ${ }^{6}$. La extensa formulación que se lee en la carta a Tito es también muy semejante en cuanto al contenido y la finalidad.

La superscriptio de 1. y 2 Corintios es muy semejante, y en ambas relativamente breve; San Pablo se presenta como apóstol; en 1 Cor, claramente afirma haber sido "llamado": la vocación al apostolado es, en verdad, el acontecimiento oficial que da la orientación definitiva a su vida y que lo define ante Cristo y ante la finalidad de su posterior misión. Al presentarse como "llamado" se percibe la intención de poner en evidencia que el agente de esa acción es Jesucristo; fue él quien intervino en su vida y cambió radicalmente toda su existencia. La relación básica de la llamada y de su condición de apóstol es con Cristo Jesús. En esta parte de estas dos cartas encontramos la fórmula sintética que todo lo relaciona con Dios y su designio: "Por voluntad de Dios". No se trata de una adición casual ni menos aún superficial: la identificación como apóstol trae para los destinatarios de la misión de Pablo la presencia y acción del Resucitado, pero en él trae al mismo tiempo la presencia y la acción de Dios. Como acompañante de Pablo menciona en la superscriptio de 1 Cor a "Sóstenes, el hermano", y en 2 Cor a "Timoteo, el hermano".

${ }^{6}$ Generalmente, los estudiosos y comentaristas relacionan muy estrechamente la llamada del apóstol con la voluntad de Dios: este énfasis subraya la iniciativa de Dios, su designio y la realización histórica de lo que Dios busca al elegir a san Pablo; a manera de ejemplo, se pueden consultar las obras siguientes: GNILKA, Epheserbrief, 53; ID., Kolosserbrief, 28; FITZMYER, First Corinthians, 123. 
La superscriptio de la carta a los Romanos es la más larga de todas y la más densa y sistemática desde varios puntos de vista. El apóstol, en esta carta, concentra una buena parte de su predicación; como explicación de algunas de las características de esta carta que pueden iluminar la lectura se mencionan las siguientes: por el proyecto de ir a España pasando por Roma, a san Pablo le interesa presentar -a una Iglesia no fundada por él- lo que ha sido su actuación como apóstol y su predicación, para prevenir interpretaciones inadecuadas y para promover la unidad de la Iglesia; su experiencia en todas partes se ha encontrado con la oposición de los judaizantes y con los antagonismos y divisiones que, en la práctica, se fueron presentando ${ }^{7}$. Todo esto se manifiesta en un texto muy rico de información y matices: Rom 1,1-5.

La autopresentación del apóstol tiene tres elementos; el primero formula su relación básica con Jesucristo: "Siervo de Cristo Jesús". El término "siervo" (doûlos = esclavo) en un ambiente helenista podría considerarse como un ser cuyos derechos están todos en su señor, y a veces podría tener un significado algo peyorativo; en contexto bíblico se podría iluminar con el personaje que aparece en los cuatro cánticos del Deuteroisaías, el "Siervo de Yahvé"; en este caso se debería reconocer a un ser humano elegido por Dios, muy cercano a él y a quien se le confía una misión que se armoniza con el designio salvífico de Dios. Si bien esto brinda alguna iluminación, de hecho, san Pablo no se presenta como siervo de Yahvé, sino como siervo de Cristo Jesús; en este primer elemento de su autopresentación no brinda la explicación: únicamente afirma que él, como siervo, pertenece a Cristo Jesús, que es de él. En síntesis, se puede afirmar que Pablo se considera en una muy estrecha relación con Jesucristo: él es su Señor y fue él quien se acercó a Pablo; es posible que, en el fondo, esté presente el acontecimiento que tuvo lugar en el camino de Damasco, pero no se menciona aquí una referencia explícita. En la carta a los Gálatas hace más clara la comprensión de su llamada (cf. Gal 1,15-17).

El segundo elemento, "apóstol llamado", desentraña la intervención del Resucitado en su vida y su finalidad ${ }^{9}$. De dicha intervención depende la conciencia que Pablo tiene de ser apóstol; en ningún lugar afirma pertenecer al grupo de los doce apóstoles ni se equipara estrictamente

7 Rom 15,14-33 ofrece la documentación de estas preocupaciones del apóstol.

8 "Llamado" es san Pablo; en la adscriptio se leerá que los creyentes de Roma son "llamados" por Jesucristo y que son al mismo tiempo santos "llamados".

9 Lo afirmado en la superscriptio de Gal a propósito del término "apóstol" se debe tener presente en este lugar. 
con ellos: si se presenta como apóstol, se debe a la intervención directa del Resucitado en su vida y la diferencia con el grupo de los doce se halla en lo específico de su misión: anunciar el evangelio a los gentiles. El participio "llamado" indica haber sido objeto de la intervención de quien lo llamó; el término "apóstol" define, sin precisar, el para qué de la llamada. En los lugares donde se encuentra el uso femenino del término ("llamada"), sobre todo en Lv 23, dicho término indica la comunidad del pueblo de Dios "convocada" para el culto; una traducción frecuente en español es el término "asamblea" (= "convocación"); en el uso plural del término, generalmente se indica a grupos del pueblo elegido que presentan diversos matices. Estos datos nos permiten ver que san Pablo desea compartir con sus lectores, además de su relación con Jesucristo y con Dios, su pertenencia al nuevo pueblo elegido de Dios y su función en él.

En el tercer elemento hace más explícita la finalidad de su llamada y de su misión: "Segregado para el evangelio de Dios" gregado", según su sentido, puede ser sinónimo de "santificado" "1 ; la sugerencia de la anterior nota hace ver que se trata de una actuación de Dios: quien es segregado por él llega a estar en el ámbito que es de Dios, y se suele ilustrar con el texto de Lv 20,26. La otra sugerencia que hace la misma nota tiene en cuenta la etimología: en este caso, "segregado" viene a ser sinónimo de "apartado", y se podría quizá percibir al menos una alusión al contenido de la palabra "fariseo". Pero san Pablo, que había sido fariseo, al presentarse ahora como "segregado para el evangelio de Dios", da al término un significado muy distinto: es "separado" de todo lo anterior, también de su fariseísmo, para recibir de Dios una nueva orientación de toda su existencia y ser destinado "para el evangelio de Dios". Una vez más, los datos de Gal 1,13-17 pueden ilustrar lo que se insinúa con el participio.

La realidad que hace concreta, que explica la intervención de Dios, y a la vez polariza toda la autopresentación de san Pablo recibe el nombre de "evangelio de Dios" ". Los capítulos 1-11 de la carta a los Romanos son

${ }^{10}$ Los comentarios hacen análisis muy elocuentes de estos términos y de su valor; podemos citar algunos: Lagrange, Romains, 1-4; Lyonnet, Ad Romanos, 14-24; Huby - Lyonnet, Romani, 39-45; Kuss, Römerbrief, 2-4; Michel, An die Römer, 63-69; Käsemann, An die Römer, 3-7; Schlier, Römerbrief, 18, 22; Fitzmyer, Romans, 227-232; Pongutá, Evangelio, 21-33.

11 Cf. Strack - Billerbeck, III, 4, cita a Jr 1,5; y, según la etimología, lo relaciona también con prwš.

12 Los escritos mencionados en la nota 10 ilustran ampliamente esta expresión tan central en este contexto. Respecto a la sencillez y brevedad, véase PONGUTÁ, Evangelio, 27-33. 
la exposición sistemática de lo que san Pablo entiende por dicha realidad. Por de pronto, en esta parte de la superscriptio, el texto brinda solo algunos datos esenciales que orientan al lector en la comprensión de dicha realidad. El primer dato refiere el preanuncio de Dios contenido en las Escrituras y dado por la mediación de los profetas ${ }^{13}$ : de alguna manera se percibe la unidad y el progreso de la comunicación de Dios a partir del Antiguo Testamento con lo que ahora se revela en la etapa mesiánica de la plenitud del tiempo. Lo más sorprendente de todo es tal vez la expresión "acerca de su Hijo": todo lo que en este texto se refiere a él viene a ser definición y concentración de la comprensión que el apóstol quiere compartir acerca de la expresión "evangelio de Dios". En primer lugar, y, en el fondo de todo, es un ser personal que en sentido estricto es "Hijo de Dios". De él hace san Pablo tres grandes afirmaciones: su condición histórica, su entronización a partir de la resurrección por obra del Espíritu como fuente de santificación y su identidad, que lo hace ver como la autoridad de quien recibió la gracia y el apostolado para obtener la fe de los gentiles ${ }^{14}$.

\subsection{La superscriptio en el segundo grupo de cartas (Flp; Flm; Col; Ef)}

Mencionamos únicamente algunas de las características propias de este grupo de cartas que se suelen considerar como cartas de la cautividad.

El nombre de Timoteo se agrega al de Pablo en las tres primeras; en Flp, ambos nombres se califican como "siervos de Cristo Jesús"; en Flm y en Col, el nombre de Timoteo va acompañado de la expresión "el hermano". En la carta a Filemón, Pablo se presenta como "prisionero de Cristo Jesús" (Flm 1 y 9) ${ }^{15}$. Generalmente se afirma que la prisión en la que se encuentra el apóstol cuando escribe esta carta es la de Roma. Su condición

${ }^{13}$ Los textos proféticos que mejor pueden ilustrar los antecedentes del término “evangelio" y del verbo "evangelizar" pueden ser Nah 2,1; Is 52,7-10; Is 61,1. El uso de estos términos en escritos de san Pablo es preponderante: "evangelio" se lee 76x en el NT, y de ellas 56x están en san Pablo; "evangelizar" aparece 54x en el NT, y de ellas $31 x$ en escritos paulinos. El título y el contenido total de san Marcos es “Evangelio de Jesucristo" (Mc 1,1).

14 Los datos de estas afirmaciones se hallan también artísticamente presentados, con expresiones muy semejantes, en el pasaje de Flp 2,6-11.

15 Este calificativo se lee también en otros lugares de algunas cartas paulinas: Ef 3,$1 ; 4,1 ; 2 \operatorname{Tm} 1,8 ;$ Heb 10,$34 ; 13,3$. 
de "apóstol de Cristo Jesús" se halla explícita en las cartas a los Colosenses y a los Efesios. Quizá la relación tan cercana con la Iglesia de Filipos y su relación con Filemón sean suficiente explicación para prescindir de dicha presentación: era evidente y clara para los destinatarios y no había necesidad de referirla. Al hacer explícita su condición de "apóstol de Cristo Jesús" en Col y Ef agrega la expresión "por voluntad de Dios". Fuente inmediata de su apostolado es Cristo Jesús; pero en el fondo de todo se halla el designio y el querer de Dios. Esta precisión confiere a la intervención de Pablo en su apostolado una consistencia y un valor incomparables: cuando él llega a un grupo humano a llevar el "evangelio", está representando para los destinatarios no solo a Jesucristo, sino al mismo Dios.

\subsection{La superscriptio en el tercer grupo de cartas (1 Tm; 2 Tm; Tit)}

Estas cartas se califican como Cartas Pastorales. Son ciertamente intervenciones de Pablo como persona, pero motivadas por su identidad y condición de apóstol; van dirigidas a personas individuales concretas y, sin embargo, su relación con Pablo no es solo personal: ellos están al frente de comunidades creadas por la acción evangelizadora de Pablo y están orientadas al bien de dichas comunidades eclesiales; estas intervenciones poseen algo de oficialidad.

En los textos de las tres se presenta Pablo como "apóstol", si bien, al hacerlo, se puede constatar alguna diferencia de la especificación. En $1 \mathrm{Tm}$, el apostolado se explica por "el mandato de Dios, Salvador nuestro, y de Cristo Jesús, esperanza nuestra"; en 2 Tm, el apostolado de Pablo se explica "por voluntad de Dios, según la promesa de vida que está en Cristo Jesús". Hablar de "mandato de Dios" o "por voluntad de Dios", a la hora de la verdad vienen a ser dos expresiones coincidentes. Otro tanto se puede afirmar de las otras expresiones: "Salvador nuestro [y] promesa de vida". En estas dos cartas se puede constatar muy claramente el protagonismo de Dios, principio y fuente de todo; de la misma manera, la mediación de Cristo se halla muy bien relacionada con Dios como fuente de la misma, y su función se califica como esperanza y promesa de vida.

La carta a Tito ofrece una superscriptio extensa y algo compleja:

Pablo, siervo de Dios, pero apóstol de Jesucristo para la fe de los elegidos de Dios y el reconocimiento de la verdad, la que según la piedad es para la esperanza de la vida eterna; el Dios que nunca miente la prometió antes de los siglos eternos, pero él manifestó su palabra en los tiempos oportunos por medio del kerigma, el que me fue confiado según el mandato de Dios, nuestro Salvador. 
Al presentarse Pablo como "siervo de Dios" surge la asociación y recuerdo del "Siervo de Yahvé" mencionado en los cánticos del Deuteroisaías: la función profética aparece como algo sugerido por dichos textos; pero enseguida todo se concreta en la expresión "apóstol de Jesucristo".

La finalidad del servicio confiado a Pablo, y especialmente la de su apostolado, se formula en un primer momento como "la fe de los elegidos de Dios y el reconocimiento de la verdad". Se trata de la acogida y la respuesta que todo ser humano debe hacer al recibir todo lo que el apóstol transmite de parte de Jesucristo a los destinatarios de su misión. Al mencionar a los "elegidos de Dios" no se está haciendo una restricción de los destinatarios, ya que la elección de Dios es universal y acontece precisamente en Cristo antes de la creación del mundo ${ }^{16}$. La parte que sigue y que formula la finalidad de la misión que san Pablo recibe de Cristo -el "reconocimiento de la verdad"- es expresión paralela a la anterior y supone que todo creyente reconoce que Cristo es "la verdad" (= la fidelidad, la consistencia, la certeza, propias de Dios). En este mismo contexto, la frase que sigue aclara algo más la acogida y una consecuencia de la respuesta del creyente: en él surge "la piedad", es decir, la fe vivida y la relación nueva del creyente con Dios: "La que es para la esperanza de la vida eterna". En la segunda parte de esta superscriptio de la carta a Tito ofrece el texto una secuencia que se puede relacionar con alguna aproximación a los datos del prólogo del evangelio según san Juan: leemos en primer lugar el término "promesa" (que se puede calificar como eterna) y que viene a ser sinónimo del término «palabra» manifestada, como afirma en la carta a los Gálatas (cf. Gal 4,4: "Cuando llegó la plenitud del tiempo..."), y ambas convergentes en el término kérygma. Todo lo que significa esta palabra fue lo que se le confió de parte de Dios a san Pablo ("según el mandato de Dios, nuestro Salvador").

\section{La adscriptio}

\subsection{La adscriptio (destinatarios) del primer grupo de cartas (1 Ts; 2 Ts; Gal; 1 Cor; 2 Cor; Rom)}

Las comunidades de Tesalónica, Galacia y Corinto fueron fundadas por san Pablo. La de Roma no. Como se sabe, el motivo por el cual escribe

16 Cf. Ef 1,4: kathòs exeléxato hēmâs en autọ prò katabolês kósmou, "él [Dios] nos eligió en él [Cristo] antes de la fundación del mundo". El "nosotros" que emplea aquí san Pablo no se puede referir a un grupo pequeño de elegidos. 
a esta comunidad se relaciona en el proyecto de ir a España a llevar el evangelio. El deseo de promover la unidad de los cristianos, la circunstancia de su paso por Roma y la importancia de dicha comunidad motivó quizá esta carta.

En 1 Ts y 2 Ts, la adscriptio es idéntica; sintética y simple es la de la carta a los Gálatas; amplia y rica es la de 1 Cor; semejante y abreviada es la de 2 Cor. En la carta a los Romanos se mencionan algunos títulos que describen a la comunidad, pero no se emplea el término "iglesia" con el que identifica a los miembros de las otras comunidades.

Con el término "iglesia" identifica san Pablo a las comunidades a las que escribe, y agrega generalmente el nombre de la localidad donde se hallan. Llama la atención que emplee el término desde 1 Ts, la más antigua de todas: tê ekklēsíai thessalonikéōn ("a la Iglesia de los tesalonicenses"). Este hecho confiere un colorido muy real e histórico a la intervención del apóstol y ubica muy concretamente a las comunidades en la cultura, la geografía y la historia. El término "iglesia" lleva artículo y se halla especificado no solo por un gentilicio, sino que inmediatamente se expone su ubicación teológica, según el modo de pensar de Pablo: "En Dios Padre y en el Señor Jesucristo".

Aunque ha pasado relativamente poco tiempo después de la Pascua, el uso del término "iglesia" da a entender que ya se ha logrado un cierto grado de identidad cristiana que lleva a evitar el uso del término "sinagoga", término universalmente empleado por los judíos para designar tanto el lugar de reunión como la asamblea en cuanto tal de los que se congregaban el sábado para el culto. Es posible que en la elección del término "iglesia" (ekklèsía) se refleje la intención de presentar a la comunidad de los nuevos creyentes como la realización escatológica de la llamada qahal Yhwh ${ }^{17}$. Esta expresión pone de manifiesto el hecho de la iniciativa de Dios: es él quien "llama", quien "convoca" a los israelitas, y en la plenitud de los tiempos a todos los hombres; la iniciativa formula a la vez la realidad tan importante de la elección de la que derivan tantas consecuencias que marcarán definitivamente la vida del pueblo de Dios. La respuesta a la convocación supone la fe, y la común llamada crea la comunidad de los que se reúnen alrededor de la Palabra y expresan así su pertenencia a Yahvé. Una expresión muy importante y central de la convocación y de la respuesta tenía lugar con ocasión del culto. Si san Pablo emplea el término ekklēsía, tal

17 A manera de ejemplo se puede referir una serie de expresiones que se hallan en el Deuteronomio (Dt 23,3.4.9), en la parte legislativa, que formula disposiciones para admitir o no a la asamblea de Yahvé en un contexto especialmente litúrgico. 
vez no quiere decir que solo cuando se reúnen los cristianos para el culto realizan la "Iglesia de Dios", sino que en toda circunstancia la están realizando. Lo que la antigua convocación de Dios prefiguraba y anunciaba se realiza ahora, en la plenitud de los tiempos, en la comunidad de los cristianos. Pablo, al llegar a Tesalónica, ubicó la llamada de Dios en la geografía y en la historia concretas. La respuesta de los tesalonicenses dio paso a su integración en la gran realidad de la convocación escatológica de Dios (de "la Iglesia de Dios"). Como si fuera poco, la ubicación teológica que sigue precisa y enriquece considerablemente el concepto de "Iglesia": esta se halla "en Dios Padre y en el Señor Jesucristo". Esto pone de relieve no solo la fe teológica que los cristianos comparten con los miembros del antiguo Israel, fe que subraya no solo la relación de filiación con respecto al Padre, sino que formula la novedad de la ubicación simultánea "en el Señor Jesucristo". Esto es lo específico de la era escatológica: la convocación de Dios y la respuesta de los nuevos creyentes acontecen en concreto en Cristo Jesús. Dios, Cristo e Iglesia aparecen en perfecta unidad vital.

En la carta a los Gálatas, san Pablo indica a los destinatarios como "las Iglesias de Galacia". Este plural supone la existencia de varias comunidades con la misma referencia y denominación. Una comunidad de los nuevos creyentes no agota toda la realidad del concepto "Iglesia" (ekklēsía); quizá se puede decir que cada comunidad, grande o pequeña, es una concreción geográfica e histórica de la realidad y misterio de la "Iglesia" total, que se halla siempre en construcción. Y cada "Iglesia" realiza, al mismo tiempo, el misterio grande de la Iglesia de Dios (la qahal Yhwh). Nace de la elección de Dios, del acontecimiento escatológico de la iniciativa de Dios, que convocó en él a sus elegidos y de la respuesta de fe por parte de los elegidos para llegar a ser habitación de Dios y para llegar a habitar en Dios.

Al comenzar la adscriptio en las dos cartas a los Corintios, san Pablo identifica a la comunidad destinataria de su escrito como la "Iglesia de Dios que está en Corinto". Es evidente que subraya en primer lugar la dimensión teológica de su comunidad. En segundo lugar, ubica a la comunidad en la geografía concreta: en Corinto. Al indicar la comunidad a la que escribe su carta, el apóstol resalta en primer plano todo lo que la expresión qahal Yhwh quiere significar. Ciertamente, la adscriptio de 1 Cor es algo compleja y tal vez no es necesario tratar de identificar los puntos que se juzguen extraños: lo que importa es la identificación de todo lo que san Pablo quiere poner en evidencia de dicha comunidad, bien sea con la designación "Iglesia de Dios" o con la presentación de lo que caracteriza a los miembros que la componen. La expresión "la Iglesia de Dios" manifiesta 
la totalidad y la unidad de la comunidad destinataria de la carta; por las expresiones que siguen, el lector percibe que dicha comunidad está formada por una pluralidad de personas a quienes reconoce como "santificados en Cristo Jesús". Este participio perfecto pasivo supone una acción de Dios con un fin preciso: él es quien santifica. Algunos textos del Antiguo Testamento utilizan este participio para referirlo a los sacerdotes, que son apartados del pueblo para poder servir en la liturgia del templo (cf. $2 \mathrm{Cr} 26,18$; Ez 48,11); en la relación que el apóstol Pablo hace de la misión recibida del Resucitado, entre otras cosas refiere lo siguiente: "Te libraré de tu pueblo y de los gentiles, a quienes te envío, para que les abras los ojos y se vuelvan de las tinieblas a la luz y del dominio de Satanás a Dios; para que reciban el perdón de los pecados y parte en la herencia entre los que han sido santificados por la fe en mé" (Hch 26,17-18) ${ }^{18}$. La misión del apóstol, al brindar a cada ser humano el misterio de Jesucristo -el evangelio- y al obtener la respuesta de fe en Cristo, pone en actividad el poder de santificación propio de la mediación de Cristo (cf. Rom 1,4). Esta santificación se refiere a la nueva relación del creyente con Dios: en otras palabras, lo traslada al mundo de Dios; se podría decir quizá que lo hace partícipe del ser mismo de Dios, y que lo hace también apto para dar culto a Dios (cf. Rom 12,1$2)^{19}$; mediador de la santificación es Cristo, y el culto que el creyente tributa a Dios acontece a la vez en Cristo Jesús.

San Pablo concluye la enumeración de lo que caracteriza a la comunidad de Corinto con una afirmación que, en cierta manera, estaba ya implícita en la expresión "Iglesia de Dios": los miembros de la comunidad de Corinto, además de haber sido santificados en Cristo Jesús, son también "llamados a ser santos". La santificación no se debe entender como un hecho concreto que obra Dios por medio de Jesucristo en un momento determinado de la vida del creyente o de la comunidad a la que pertenece: se debe entender como un proceso que va santificando permanentemente al creyente.

San Pablo amplía luego el horizonte e integra en la comunidad a la que escribe a "todos los que en cualquier lugar invocan el nombre del Señor nuestro, Jesucristo". Jesucristo es Señor De todos. Esta invocación viene a coincidir con el reconocimiento de la divinidad de Jesucristo y a la vez es manifestación de la total confianza puesta en él. La actitud es estrictamente religiosa y se asemeja a la actitud presente en algunos salmos en los

18 Esta idea la había presentado antes san Pablo en el llamado Discurso a los ancianos de Mileto (cf. Hch 20,32).

19 Esto lo afirma con claridad Fitzmyer, First Corinthians, 126. 
que se dice que se invoca el nombre de Dios (cf. Sal 85,5; 98,6; 144,18; cf. también SalSalomón 2,36; 9,6). Por lo demás, son muchos los lugares bíblicos en los que se menciona la invocación de Dios, comenzando por el dato de Gn 4,26 (Set). La ampliación del horizonte se encuentra de una manera semejante en la adscriptio de 2 Cor: "Con los santos todos, los que están en toda Acaya".

La adscriptio de la carta a los Romanos presenta algunas particularidades: ante todo, no utiliza la expresión "Iglesia de Dios" ni tampoco la palabra "iglesia" para indicar a la comunidad de Roma. Los datos acerca de la identidad y características de la comunidad a la que escribe son muy significativos. El primero se encuentra en la conclusión de la autopresentación del apóstol: "Por él recibimos gracia y apostolado para obediencia de fe entre todos los gentiles por su nombre". Esta viene a ser la exposición de la fuente que explica la intervención actual de san Pablo ante la comunidad de Roma, la finalidad y los destinatarios específicos de la misión recibida del Resucitado. En este contexto tiene mucha importancia la mención de los gentiles como destinatarios de la misión de san Pablo. Esta afirmación, entre otras cosas, le sirve de puente para dirigirse a la comunidad de Roma: entre los miembros actuales de la comunidad hay también creyentes que, antes de serlo, fueron gentiles, y puede haber gentiles que están en proceso de integración: estos hechos explican la intervención del apóstol por medio de su carta. Independientemente de las estadísticas o de la proporción de los miembros provenientes de la gentilidad que forman parte de la comunidad de Roma, lo que es decisivo para explicar esta intervención de san Pablo es la afinidad de su misión con la composición de dicha comunidad. San Pablo generaliza la composición de la comunidad como si todos los actuales miembros hubieran sido gentiles: "Entre ellos [gentiles] estáis también vosotros".

A continuación precisa san Pablo con tres calificativos las características de los destinatarios de su carta: todos son "llamados de Jesucristo, amados de Dios, llamados santos". Todos y cada uno de los miembros son "llamados de Jesucristo": esta expresión hace las veces de la identificación como Iglesia o Iglesia de Dios. La concreción histórica en la plenitud del tiempo de la convocación de Dios acontece en Jesucristo. Hablar de Iglesia de Dios o Iglesia de Jesucristo viene a ser lo mismo. Si los miembros de la comunidad de Roma son "amados de Dios", ellos no solo son objeto del amor de Dios, sino que deben saber que ahora forman parte del pueblo elegido de $\operatorname{Dios}^{20}$. Si, además, los miembros de la comunidad de

${ }^{20}$ El texto de Dt 7,7-8 ilustra esta afirmación. 
Roma son "llamados santos", no solo se indica el impulso vital que debe animar a todos los miembros de la comunidad en todo tiempo y circunstan$\operatorname{cia}^{21}$, sino también la meta definitiva de toda la vida del creyente.

El primero de los tres calificativos con los que san Pablo menciona a sus destinatarios de Roma los relaciona con Jesucristo; los otros dos los relacionan directamente con Dios. La llegada del evangelio (= Jesucristo) a Roma fue también la expresión especial del amor de Dios a los creyentes; pero la llamada de Jesucristo y el amor de Dios los hicieron miembros del pueblo nuevo de Dios, pueblo llamado a participar de la santidad misma de Dios. El término "santo" posee una densidad misteriosa y grande de significado: su uso en el Antiguo Testamento es muy rico y variado. En la narración de la vocación de Isaías, los serafines proclaman tres veces dicho término a modo de un superlativo enfático y lo refieren a $Y h w h S^{e} b \bar{a}$ 'ot . El es el Santo por esencia y por excelencia (cf. Is 6,3). Se emplea además para expresar la muy especial relación que lugares, personas o tiempos pueden tener con Dios: el lugar santo (Lv 6,9.19.20; 7,6, etc.; Ex 3,5); los sacerdotes ( $\operatorname{Lv} 21,8)$; un nazir (Nm 6,9); toda la comunidad de Israel (Nm 16,3); es un deber de todo Isael ( $\mathrm{Lv} 19,2)$; califica de un modo especial al pueblo elegido de Dios (Dt 7,6; 14,21, etc.). En Lv 23 se presenta el calendario de las solemnidades litúrgicas de Israel, a las que se les da el nombre de "asamblea santa" (= convocación). Los antecedentes del Antiguo Testamento vienen a ser un trasfondo que ilustra muy bien y explica el uso del término "santo" para indicar con él a los miembros del nuevo pueblo de $\operatorname{Dios}^{22}$.

\subsection{La adscriptio (destinatarios) del segundo grupo de cartas (Flp; Flm; Col; Ef)}

Tres de estas cartas están dirigidas a comunidades; solo la de Filemón se concentra en este destinatario. San Pablo, al dirigirse a estas comunidades, no emplea el término "iglesia" ni la expresión "Iglesia de Dios". Por dirigirse a comunidades concretas, el apóstol indica el lugar geográfico de cada una: Filipos, Colosas, Éfeso. La identidad de los miembros de estas comunidades se formula con un sustantivo: "los santos". Esta identidad puede ir acompañada de algunas precisiones: "todos" (Flp), "en Cristo Je-

21 Cf., a manera de ejemplo, Lv 19,2.

22 La obra de S. Lyonnet, Exegesis epistulae ad Romanos, cap. I ad IV, 26-30, ilustra este título dado a los cristianos. 
sús" (Flp), "hermanos" ( $\mathrm{Col})$, "creyentes en Cristo" $(\mathrm{Col})$, "creyentes en Cristo Jesús" (Ef). En la carta a los Filipenses integra explícitamente a personas que tienen alguna función en la vida de la comunidad: "obispos" (syn episkópois), "diáconos". El uso del término "santos" para identificar a los miembros de estas comunidades supone ya un camino muy largo en el que se ha reconocido y verificado la obra santificadora de Dios en Cristo, que ha creado una nueva y maravillosa relación de cada creyente con Dios. Esta misma obra de Dios ha creado una relación muy cercana de todos los miembros de las comunidades: en la carta a los Colosenses, san Pablo los llama "hermanos".

La adscriptio de la breve carta a Filemón agrega al nombre del destinatario dos adjetivos: uno formula la relación de afecto que une a Pablo con la persona a quien escribe ("amado, querido"), y el otro pone de relieve la colaboración de Filemón con la obra de Pablo: "colaborador nuestro". En cierta manera, ya desde el comienzo de la carta, san Pablo crea un ambiente de acogida para el favor que le va a pedir a Filemón en favor de Onésimo.

\subsection{La adscriptio (destinatarios) del tercer grupo de cartas (1 Tm; 2 Tm; Tit)}

Los destinatarios de estas tres cartas se hallan al frente de comunidades de creyentes por encargo recibido del mismo apóstol. Sin embargo, en la adscriptio, lo único que se pone en evidencia es la relación que une a san Pablo con cada uno de ellos. En los tres casos, el término que formula dicha relación es "hijo". Se trata de una relación muy familiar y muy elocuente. En 1 Tm y en Tit se califica el término con el adjetivo "genuino", y en $2 \mathrm{Tm}$ el calificativo es "amado". Desde luego se debe entender una filiación que no es biológica, pero que, a pesar de no serlo, es algo muy profundo y fuerte. El término "amado" manifiesta además la relación interpersonal de afecto. La relación especial de san Pablo con sus colaboradores (paternidad-filiación) tiene una fuente de la cual procede y se nutre: "la fe" (1 Tm), "la común fe" (Tit). Quien lleva el don de Dios -el Hijo de Diosen todo el proceso de la evangelización a una persona o a una comunidad representa de alguna manera la paternidad de Dios; cuando los destinatarios de ese don lo reciben con el corazón y se deciden a creer, surge la especial relación con Dios y con quien en su nombre transmite el don: esta relación que resulta del encuentro del don y de la fe produce la filiación divina. 


\section{El saludo (salutatio) en las cartas de san Pablo}

En la cultura tanto griega como romana la parte introductoria del epistolario -la praescriptio- incluía en el tercer lugar el saludo que quien escribía la carta hacía a su destinatarios o destinatarios. Se trataba de una fórmula generalmente muy breve que transmitía una especie de augurio. Los de cultura helenista empleaban el verbo jaírein (salud o alegrarse); los latinos, el término ave, y los semitas, el término šālôm (paz) ${ }^{23}$. El saludo epistolar refleja las costumbres de las relaciones interpersonales, en las que se podían verificar los mismos términos tanto para saludar como para despedirse después de un encuentro: jaire o jaírein en la cultura helenista y šălôm en el mundo israelita. En el género epistolar del mundo griego parece prevalecer el uso del verbo jaírein; algunos ejemplos de este uso en libros relacionados con la Biblia pueden ser: Esdras (apócrifo) 6,8; 8,9; Est 8,12; en el NT se puede documentar en Hch 15,23; 23,26; Sant 1,1. El término empleado en el saludo y en el género epistolar en la cultura israelita ( $\breve{s}$ lôm) augura el bienestar, la tranquilidad completa en todo, y se suele traducir con nuestro vocablo "paz"; pero puede ofrecer muchos matices que dependerán del contexto en el que se emplee. Las diversas culturas semitas tienen términos muy semejantes al empleado por los israelitas ${ }^{24}$.

La salutatio en las cartas de san Pablo contiene algunas particularidades: mencionamos en primer lugar un núcleo que se lee en todos los saludos como algo casi estereotipado: "Gracia a vosotros y paz" (járis hymîn kaì eiréne). En segundo lugar, a este núcleo agrega san Pablo, con excepción del saludo empleado en $1 \mathrm{Ts}$, otros elementos que lo enriquecen de diversas maneras; en las cartas a Timoteo, por ejemplo, adjunta el término "misericordia". La amplificación más notoria es la de la carta a los Gálatas. La presencia de los dos términos en lo que hemos llamado núcleo del saludo no parece que se pueda explicar por el solo deseo de tener en cuenta tanto la cultura griega como la semita. Como fuente inspiradora de la utilización de los términos "gracia" y "paz" se ha sugerido el texto de la bellísima bendición que se lee en Nm 6,22-26; de hecho, en la bendición que Aarón debía emplear para bendecir al pueblo se encuentran esos dos térmi-

${ }^{23}$ Lagrange hace una referencia al 2 Mac $(1,1)$ : en este saludo se encuentra, en práctica, la costumbre griega y la semita: el verbo jaírein y el augurio de la paz (eirếne agathēn), cf. LAgRAnge, Epître aux Romains, 11.

${ }^{24}$ A manera de ejemplo se puede citar la obra de CunchiLlos, Epistolografía ugarítica. Se puede constatar el uso de la raíz šlm en el saludo de algunas de las cartas estudiadas (cf. especialmente los textos KTU 2.11 y KTU 2.12). 
nos: el término "gracia" no se lee directamente en la traducción de los LXX, pero está sugerido por el verbo hebreo ḥnn (wîhunnékā); el término "paz" sí está presente de manera explícita ${ }^{25}$. San Pablo, profundo conocedor del AT, no necesitaba acudir a la cultura griega para la formulación de su saludo; probablemente, al utilizar el término "gracia" (járis) era sí consciente de integrar en su saludo esa antigua fórmula de bendición que podía contribuir a la ubicación en la historia de la salvación y a proclamar su plenitud en la era escatológica. De hecho, la utilización del término "gracia" en vez del verbo jaírein debió de asumirla san Pablo muy deliberadamente, porque el contenido del término aparece muy claro en textos como Rom 3,22-25.

El plural del núcleo ("vosotros"), como algo curioso, pone en evidencia lo estereotipado de la fórmula; se lee también en la carta a Filemón.

\subsection{El saludo (salutatio) en el primer grupo de cartas (1 Ts; 2 Ts; Gal; 1 Cor; 2 Cor; Rom)}

En este primer grupo de cartas, el núcleo del saludo es el ya mencionado: "Gracia a vosotros y paz". Casi en todas las cartas el núcleo presenta adiciones; la más abundante está en la carta a los Gálatas.

L. Alonso Schökel distingue en el término hẹen (= járis) dos posibles significados: gracia-belleza y gracia-favor; menciona luego algunos matices: belleza, hermosura, gracia, simpatía, atractivo, encanto; adorno, ornato; favor, estima, gracia; afecto, atracción, gusto, complacencia; agrado, caridad; amor y misericordia pueden ser sinónimos ${ }^{26}$.

La obra de Rigaux citada en la nota 25 ofrece un estudio cuidadoso y casi exhaustivo de los términos "gracia" y "paz". Menciona el uso del término "gracia" en el griego profano, en los LXX, en el NT y especialmente en san Pablo. Por su interés referimos algunas de sus anotaciones.

Con el término járis, según Rigaux, los LXX traducen formas hebreas como las siguientes: hẹen (Prov 22,1: "gracia, favor"); pen-yehassedkā

${ }^{25}$ Aarón debe bendecir al pueblo con estas palabras: ${ }^{24}$ eulogếsai se kýrios kai fyláxai se ${ }^{25}$ epifánai kýrios tò prósōpon autoû epi sè kai eleếsai se ${ }^{26}$ epárai kýrios tò prósōpon autoû epi sè kai dô(i)ẽ soi eirếne. El texto hebreo ofrece términos muy significativos: šmr, hnnn, śym šălôm + le. Para el contenido de los términos "gracia" y "paz", y para la sugerencia, se puede consultar la obra de RIgAux, Saint Paul, 352355. Sobre estos términos hay muchos estudios aislados o como parte de comentarios; cf., por ejemplo, el artículo de Stoebe, “nנן, (hnn)”, 587-597.

${ }^{26}$ Cf. Alonso Schökel, S. v. 
(Prov 25,10: $h s d=$ "tener misericordia"); tôb (Prov 18,22: "un bien, beneficio"); rah̆ămîm (Gn 43,14: "compasión, favor"); rāṣôn (Prov 10,32: "agrado, complacencia"). Hay, por tanto, una pluralidad de matices de significado que no se podrá eliminar, sino tratar de precisar en cada caso cuál o cuáles de esos matices se deben tener en cuenta.

Una observación muy generalizada considera que el uso del término járis en el saludo es muy propio de san Pablo. El análisis que hace Rigaux de la presencia de este término en el NT se concentra principalmente en algunos pasajes de los Hechos de los Apóstoles y en textos de los escritos de san Pablo. Los más pertinentes serían: Hch 15,40: "Entregado por los hermanos a la gracia del Señor"; Hch 20,32: "De donde fueron entregados a la gracia de Dios a la obra que habían completado". En ambos lugares el verbo "entregar" se refiere a la misión que Pablo y Silvano recibieron de la Iglesia de Antioquía para anunciar el evangelio (la realización de la promesa presente en escritos proféticos para la era mesiánica, la persona y la obra salvífica de Jesucristo) a las ciudades de Asia Menor en donde se podían encontrar grupos humanos judíos y con ellos también paganos. Realizar esa misión era ser entregados, dedicados, a la gracia del Señor o, lo que es lo mismo, a la gracia de Dios. En Rom 3,24 se lee: "Justificados gratuitamente por su gracia, por la redención que está en Cristo Jesús" ${ }^{27}$. La gracia del Señor, o la gracia de Dios, es entonces el acontecimiento escatológico de salvación concentrado en Cristo Jesús. Ante la constatación del uso del término járis en el saludo de san Pablo a sus destinatarios, acompañado del término "paz", o de "misericordia" en las pastorales, y teniendo en cuenta los datos del libro de los Hechos, Rigaux hace la siguiente consideración: "Para él [san Pablo], el favor divino se manifestó de manera superabundante en la universalidad de la salvación brindada a los paganos, en la liberalidad del favor divino al extenderse sin consideración de personas y en la libertad con la cual Dios distribuye sus dones, perdonando a los pecadores judíos y gentiles, dándoles la plenitud de la vida nueva en Cristo y en el Espíritu; cf. Rom 5,15; 16,20; 1 Cor 1,4; 2 Cor 4,$15 ; 6,1 ; 9,8 ;$ Ef 4,7.29" ${ }^{28}$.

27 Según este pasaje, los seres humanos que eran pecadores tienen ahora la experiencia de la justificación como algo recibido gratuitamente; la eficiencia de la justificación la atribuye san Pablo a la gracia de Dios; la gracia de Dios, por su parte, actúa por medio de la redención que está en Cristo Jesús. El contenido de este texto puede iluminar muy bien lo que el apóstol desea a los destinatarios de sus cartas en el saludo. Para una síntesis de la noción de redención (apolýtrôsis), cf. Pongutá, Evangelio, 172-177.

28 Rigaux, Saint Paul, 353. 
Como hemos señalado, la segunda palabra del núcleo del saludo propio de las cartas paulinas es eirênē y se suele traducir con el término "paz" ${ }^{29}$. Por las características de los escritos del apóstol, lo que él desea en su saludo no debe limitarse al solo augurio de tranquilidad, bienestar, plena serenidad o cosa parecida; tampoco se puede reducir a la armonía entre los miembros de sus comunidades; con toda probabilidad se debe tener en cuenta el contenido de la paz propia de la era mesiánica, de los últimos tiempos, la anunciada en algunos textos proféticos (p. ej., Is 2,1-5; 9,5, etc.), la que manifiesta la armonía de las relaciones de los creyentes con Dios y, especialmente, la que se halla implícita en el saludo del Resucitado a los discípulos (cf. Jn 20,19.26).

Todas las cartas de este grupo, menos 1 Ts, presentan una ampliación del núcleo del saludo: "De parte de Dios, Padre [nuestro], y del Señor Jesucristo". La función clara de esta adición es la de indicar la fuente, el origen tanto de la gracia como de la paz, consecuencia lógica de la misma. Esta precisión permite captar la dimensión teológica y la mediación cristológica de lo que san Pablo desea a sus comunidades. La gracia y la paz que provienen de Dios Padre acontecen por medio de Jesucristo, el Señor: su eficacia y su acogida es lo que san Pablo augura en las cartas que escribe a sus Iglesias. Paralelamente se pone en evidencia el don especial de la filiación divina, derivado de la gracia y de la paz, que ahora enriquece a los miembros de los destinatarios de las cartas ${ }^{30}$. Muy importante es también el título "Señor" (kyríos) con el que acompaña el nombre de Jesucristo. Con este título se tiene en cuenta la resurrección y la profundísima relación de Jesucristo con Dios Padre ${ }^{31}$.

En el saludo del apóstol a las comunidades de Galacia se lee la anterior ampliación: esta ya sería un presupuesto para las observaciones que hará en su carta. La adición que sigue refuerza este presupuesto con afirmaciones que ponen muy en evidencia el protagonismo de Jesucristo en la obra redentora. Se trata del acontecimiento fundamental en el que Jesucristo "se entregó a sí mismo por nuestros pecados, a fin de arrancarnos de este mundo perverso" $(\mathrm{Gal} 1,4)^{32}$. Pero, precisamente este acontecimiento tuvo

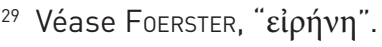

${ }^{30}$ Implicada en la expresión patròs [pátēr] hēmôn, "Padre de nosotros" = "Padre nuestro".

31 En los LXX, el título kýrios se emplea para indicar a Dios; en ocasiones se lee la expresión explícita kýrios ho theòs (desde Gn 2,8 en adelante; muchas veces se usa solo el término kýrios).

$32 \mathrm{H}$. Schlier refiere otros lugares de textos paulinos en los que se lee la idea de la entrega de Cristo con expresiones semejantes o paralelas (cf. 1 Tm 2,6; Tit 2,14; 
lugar "según la voluntad de Dios y Padre nuestro" (Gal 1,4): es el Dios y Padre nuestro quien está cumpliendo en Cristo sus antiguas promesas y actuando en la era escatológica su voluntad redentora. Como consecuencia, para las comunidades de Galacia y para todos los seres humanos, la fe y la esperanza de salvación se concentran ahora en la obra y la persona de Jesucristo. La doxología con la que concluye san Pablo su saludo sella muy bien la autenticidad teológica de su fe y del evangelio que había anunciado antes a los gálatas: "A él la gloria por los siglos de los siglos. Amén".

\subsection{El saludo (salutatio) en el segundo grupo de cartas (Flp; Flm; Col; Ef)}

El saludo que envía san Pablo en estas cartas de la cautividad conserva en gran parte la formulación estereotipada que se constató en el primer grupo: "Gracia a vosotros y paz de parte de Dios, Padre nuestro, y del Señor Jesucristo". Las peculiaridades que se pueden observar son estas: en la carta a Filemón, no obstante el hecho de ser dirigida a una persona, se lee el plural propio de las cartas dirigidas a los miembros de una comunidad eclesial ("a vosotros"); el plural reaparece también en el v. 22 y en el saludo de despedida del v. 23. Otra peculiaridad notable es una omisión en el saludo dirigido a los miembros de la comunidad de Colosas: no se lee la expresión conclusiva "y del Señor Jesucristo". La primera peculiaridad se puede explicar quizá precisamente por el carácter estereotipado de este tipo de formulaciones; para la segunda no hay una explicación fácil, pero la carta presenta contenidos que estarían en la línea perfecta de lo que se omitió. El breve comentario explicativo realizado para el saludo del primer grupo de cartas, por su coincidencia, vale también para este grupo.

\subsection{El saludo (salutatio) en el tercer grupo de cartas (1 Tm; 2 Tm; Tit 1,4)}

En las llamadas cartas pastorales se conserva en parte el carácter estereotipado del saludo. La formulación empleada en las dos cartas a Timoteo es idéntica: "Gracia, misericordia, paz, de parte de Dios Padre y de

Ef 5,2.25; Rom 4,25;8,32; 1 Cor 11,23); un aporte muy relevante es la cita del cuarto cántico del Siervo de Yahvé como antecedente de la figura y la obra de Jesucristo (Is 53,6.12); cf. Schlier, Galati, 34. 
Cristo Jesús, nuestro Señor". Aparece el término "misericordia", que no se había leído en ninguna de las cartas de los grupos anteriores. Lo llamativo del saludo en la carta a Tito es que omite el término "misericordia", prescinde del plural ("a vosotros") y cambia el título dado a Cristo Jesús en las otras cartas ("nuestro Señor") por el de "nuestro Salvador".

El término "misericordia" (éleos) se encuentra en 19 textos del NT (de las 279 veces de toda la Biblia); es la traducción que los LXX ofrecen del sustantivo hesed. Cuando se dice de Dios, tomado como una especie de adjetivo, manifiesta su bondad, su disposición para acercarse especialmente al pecador para manifestarle su solidaridad, su compasión y la eficacia de su amor que perdona y restituye su correcta relación con él. Es muy notoria la presencia de este término en el libro de los Salmos (p. ej. Sal 136; cf. Ex 34,6). Una forma emparentada con misericordia se halla en el texto de la fórmula de bendición de Nm 6,25: kaì eleésai se = "y te conceda su favor" = "y tenga misericordia de ti"). Conviene recordar que el término "misericordia" se halla con frecuencia asociado a otros que le brindan matices especiales: compasión, fidelidad, compromiso de la alianza. Estos matices se pueden suponer presentes en el saludo de san Pablo a los destinatarios de sus escritos. El término puede usarse también en el ámbito de relaciones entre seres humanos: indicaría la actitud de solidaridad, cercanía, compasión con un ser necesitado a quien se auxilia y se apoya para hacer propio su dolor y aliviarlo. Pero, por la precisión que se lee en el saludo del apóstol ("de parte de Dios Padre y de Cristo Jesús, nuestro Senor"), la misericordia que augura es la de Dios para sus destinatarios.

Si en el saludo de la carta a Tito se lee que la gracia y la paz que san Pablo le desea proviene también "de Cristo Jesús, nuestro Salvador", se debe reconocer la íntima y misteriosa unión de Dios Padre con la persona y la misión dada a Jesucristo. De ambos se afirma el título "Salvador". El contenido de este término orienta hacia una comprensión de la salvación escatológica que se puede ilustrar con expresiones de san Pablo como las que emplea para indicar la función del término "evangelio" (cf. Rom 1,16: eis sōtērían pantì tôi pisteúonti, "para la salvación de todo el que cree").

\section{Bibliografia}

Alonso SchöKel, L., Diccionario bíblico hebreo-español, Madrid 1994. Brown, R. E., An Introduction to the New Testament (ABRL), New York 1997. 
Cunchillos Ilarri, J. L., Estudios de epistolografía ugarítica, Valencia 1989.

Fitzmyer, J. A., First Corinthians (Anchor Bible), New York 2008.

-, Nuevo Comentario Bíblico San Jerónimo. Nuevo Testamento, "Gálatas", Estella 2004.

-, Romans (Anchor Bible), New York 1993.

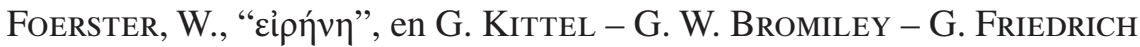
(eds.), Theological Dictionary of the New Testament 2, Grand Rapids, MI 1964, 400-402 [electronic edition]. La sección que estudia el término

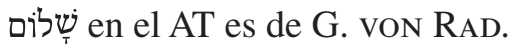

GNILKa, J., Der Epheserbrief, Freiburg 1971.

-, Der Kolosserbrief, Freiburg 1980.

Huby, J. - Lyonnet, S., San Paolo. Epistola ai Romani, Roma 1961.

Käsemann, E., An die Römer (HNT), Tübingen 1973.

Kuss, O., Der Römerbrief, Regensburg 1963.

Lagrange, M.-L., Saint Paul, Épitre aux Romains (Études Bibliques), Paris 1950.

Lyonnet, S., Exegesis epistulae ad Romanos, Roma, 1963.

Michel, O., Der Brief an die Römer, Göttingen ${ }^{5} 1978$.

Mussner, F., Der Galater Brief (HThKNT), Freiburg 1974.

Pongutá H., S., El Evangelio de Dios, Bogotá ${ }^{2} 2011$.

Rigaux, B., Saint Paul. Les Épitres aux Thessaloniciens (Études Bibliques), Paris 1956.

Schlier, H., Lettera ai Galati, Brescia 1965.

-, Der Römerbrief (HThKNT), Freiburg 1977.

Stoebe, H. J., "חנן (hnn), gnädig sein”, en E. Jenni - C. Westermann (eds.), Theologisches Handwörterbuch zum Alten Testament I, München 1971, 587-597.

[recibido: 21/08/18 - aceptado: 17/10/18] 